\title{
SLOW DIFFUSIVE GRAVITATIONAL INSTABILITY BEFORE DECOUPLING
}

\author{
ToDD A. THOMPSON ${ }^{1}$ \\ Department of Astronomy and Center for Cosmology \& Astro-Particle Physics, The Ohio State University, Columbus, OH 43210, USA; \\ thompson@astronomy.ohio-state.edu \\ Received 2009 August 12; accepted 2009 November 16; published 2010 January 12
}

\begin{abstract}
Radiative diffusion damps acoustic modes at large comoving wavenumber ( $k$ ) before decoupling ("Silk damping"). In a simple WKB analysis, neglecting moments of the temperature distribution beyond the quadrupole (the tightcoupling limit), damping appears in the acoustic mode as a term of order $i k^{2} \dot{\tau}^{-1}$, where $\dot{\tau}$ is the scattering rate per unit conformal time. Although the Jeans instability is stabilized on scales smaller than the adiabatic Jeans length, I show that the medium is linearly unstable to first order in $\dot{\tau}^{-1}$ to a slow diffusive mode. At large comoving wavenumber, the characteristic growth rate becomes independent of spatial scale and constant: $\left(t_{\mathrm{KH}} a\right)^{-1} \approx\left(128 \pi G / 9 \kappa_{T} c\right)\left(\rho_{m} / \rho_{b}\right)$, where $a$ is the scale factor, $\rho_{m}$ and $\rho_{b}$ are the matter and baryon energy density, respectively, and $\kappa_{T}$ is the Thomson opacity. This is the characteristic timescale for a fluid parcel to radiate away its total thermal energy content at the Eddington limit, analogous to the Kelvin-Helmholz (KH) timescale for a radiation pressure-dominated massive star or the Salpeter timescale for black hole growth. Although this mode grows at all times prior to decoupling and on scales smaller than roughly the horizon, the growth time is long, about 100 times the age of the universe at decoupling. Thus, it modifies the density and temperature perturbations on small scales only at the percent level. The physics of this mode in the tight-coupling limit is already accounted for in the popular codes CMBFAST and CAMB, but is typically neglected in analytic studies of the growth of primordial perturbations. The goal of this work is to clarify the physics of this diffusive instability in the epoch before decoupling, and to emphasize that the universe is formally unstable on scales below the horizon, even in the limit of very large $\dot{\tau}$. Analogous instabilities that might operate at yet earlier epochs are also mentioned.
\end{abstract}

Key words: cosmic background radiation - cosmology: theory - large-scale structure of universe

\section{INTRODUCTION}

In standard analytic treatments of perturbation theory in the epoch near radiation-matter equality the tight-coupling approximation is employed, which amounts to neglecting moments of the temperature distribution beyond the quadrupole. In this limit, the scattering rate per unit conformal time $\dot{\tau}$ is very large and, neglecting gravity at large comoving wavenumber, the dispersion relation for acoustic modes is easily derived; the medium supports stable acoustic oscillations modified by radiative ("Silk") damping on small scales (Silk 1967, 1968; Peebles \& Yu 1970; Weinberg 1971; Ma \& Bertschinger 1995; Hu \& Sugiyama 1995a, 1995b, 1996; Hu et al. 1997; Dodelson 2003). In the WKB approximation, the damping term enters as a complex correction to the acoustic mode frequencies that is $\propto k^{2} / \dot{\tau}$, becoming increasingly important at small spatial scales (see, e.g., Dodelson 2003, Section 8.4).

Most analytic calculations of radiative damping of acoustic modes neglect gravity at large comoving wavenumber, $k$, because the frequency of acoustic oscillations $\left(\omega \approx c_{s} k\right.$, where $c_{s}$ is the sound speed) is large and the medium is Jeans stable. In this paper, I show that on scales smaller than the Jeans length the medium is linearly unstable to a slow diffusive mode that is of order $\dot{\tau}^{-1}$. Thus, on small scales where the medium is dynamically Jeans stable and supports stable (but radiatively damped) acoustic oscillations, it is unstable to an orthogonal diffusive mode. This mode was earlier discussed by Yamamoto et al. (1998). The physics of this slow diffusive mode is already included in CMB codes such as CMBFAST and CAMB, which include gravity on small scales in the tight-coupling limit in their solution to the coupled equations for perturbations, and in earlier work

\footnotetext{
1 Alfred P. Sloan Fellow.
}

employing full numerical solutions to the coupled perturbation equations. However, the physics of this mode has been often neglected in analytic studies of the growth of perturbations, and for this reason a more complete discussion is warranted; it may affect analytic estimates for the characteristic baryon-acoustic oscillation (BAO) scale, the physics of low-mass dark matter halos, and the transfer function.

In Section 2, I review the physics of acoustic modes and radiative damping in the cosmological context, and I show that the unstable diffusive mode appears in a simple WKB treatment that includes gravity on small scales. The discussion is related to that of the "terminal velocity" mode presented in Yamamoto et al. (1998). I emphasize that the growth of this mode is not tied to the breakdown in the coupling between gas and radiation; indeed, it appears at the same order in $\dot{\tau}$ as Silk damping, and a temperature difference between the radiation and the gas is not required for growth. I note that an analogous mode should operate in the epoch of neutrino decoupling, or whenever the medium is partially supported against self-gravity by a relativistic and diffusing particle species. Section 3 presents a complimentary analysis in an expanding background closest in spirit to Silk (1967), and to the discussion of adiabatic modes in standard textbooks. In Section 4, I provide a brief summary.

\section{ACOUSTIC MODES AND SILK DAMPING}

Following $\mathrm{Hu} \&$ Sugiyama (1996), the equations for the evolution of the moments of the photon temperature distribution $\Theta_{n}(n=0,1,2)$ in the tight-coupling limit are

$$
\begin{gathered}
\dot{\Theta}_{0}+k \Theta_{1}+\dot{\Phi}=0 \\
\dot{\Theta}_{1}+k\left(\frac{2}{3} \Theta_{2}-\frac{1}{3} \Theta_{0}\right)-\dot{\tau}\left(\Theta_{1}-\frac{i v_{b}}{3}\right)-\frac{k \Psi}{3}=0
\end{gathered}
$$




$$
-\frac{2 k}{5} \Theta_{1}-\frac{9}{10} \dot{\tau} \Theta_{2}=0,
$$

where $v_{b}$ is the baryon velocity and the last expression neglects $\dot{\Theta}_{2}$. The continuity and Euler equations for the baryons and dark matter are

$$
\begin{gathered}
\dot{\delta}_{b}+i k v_{b}+3 \dot{\Phi}=0 \\
\dot{v}_{b}+\frac{\dot{a}}{a} v_{b}-\frac{\dot{\tau}}{R}\left(v_{b}+3 i \Theta_{1}\right)+i k \Psi=0 \\
\dot{\delta}_{m}+i k v_{m}+3 \dot{\Phi}=0 \\
\dot{v}_{m}+\frac{\dot{a}}{a} v_{m}+i k \Psi=0
\end{gathered}
$$

respectively, where $R=3 \rho_{b} / 4 \rho_{r}$,

$$
\dot{\tau}=d \tau / d \eta=-n_{e} \sigma_{T} a \approx-\rho_{b} \kappa_{T} a,
$$

$\eta$ denotes conformal time, $\kappa_{T}$ is the Thomson opacity, and the notation of Dodelson (2003) has been adopted. The field equations can be written as

$$
k^{2} \Phi+3 \frac{\dot{a}}{a}\left(\dot{\Phi}-\Psi \frac{\dot{a}}{a}\right)=4 \pi G a^{2}\left(\rho_{b} \delta_{b}+\rho_{m} \delta_{m}+4 \rho_{r} \Theta_{0}\right)
$$

and

$$
k^{2}(\Phi+\Psi)=-32 \pi G a^{2} \rho_{r} \Theta_{2},
$$

where $\rho_{b}, \rho_{m}$, and $\rho_{r}$ denote the baryon, matter, and radiation energy density, respectively. The approximate equality in Equation (8) assumes that one consider epochs sufficiently before recombination that the universe is fully ionized. The above set of equations neglects moments of the temperature distribution above the quadrupole, the polarization hierarchy, and neutrinos.

In the simplest derivation of Silk damping, one neglects both the gravitational terms and the expansion of the universe (so that $\dot{a} / a=\dot{\Phi}=\Phi=\Psi=0$ ) as well as the matter. Expanding the time dependence as $e^{i \omega t}$, one finds that

$$
\begin{aligned}
\omega\left[\omega^{3}\right. & \left.+i \dot{\tau} \omega^{2}\left(1+\frac{1}{R}+\frac{8 k^{2}}{27 \dot{\tau}^{2}}\right)-\omega \frac{k^{2}}{3}\left(1+\frac{8}{9 R}\right)-i \dot{\tau} \frac{k^{2}}{3 R}\right] \\
& =0 .
\end{aligned}
$$

Expanding to lowest order in the limit of large $\dot{\tau}$, there are two acoustic modes

$$
\omega_{\text {acoustic }} \approx \pm c_{s} k-\frac{i k^{2}}{2(1+R) \dot{\tau}}\left(c_{s}^{2} R^{2}+\frac{8}{27}\right),
$$

where $c_{s}^{2}=c^{2} /[3(1+R)]$, and the purely damped mode (see, e.g., Blaes \& Socrates 2003) ${ }^{2}$

$$
\omega_{\text {damped }} \approx-i \dot{\tau}\left(\frac{1+R}{R}\right)-\frac{i k^{2} R}{27 \dot{\tau}} \frac{(8-R)}{(1+R)^{2}} .
$$

Because $\dot{\tau}$ is always negative and because I have written the time dependence as $e^{i \omega t}$, all terms in all three modes involving $\dot{\tau}$ represent damping. Note that Equation (11) contains a fourth mode that has $\omega=0$, which I now discuss when gravity is included in the system.

\footnotetext{
2 Note that $\omega_{\text {damped }}$ disappears from the analysis if the gas and radiation temperatures are assumed to be equal, as in Section 3 (e.g., Blaes \& Socrates 2003; Kaneko \& Morita 2006; Appendix B of Thompson 2008).
}

\subsection{The Simplest Case with Gravity}

The neglect of gravity in the above derivation is justified on small scales for acoustic modes whose frequencies are $\propto c_{s} k$ (Equation (12)). It turns out that it is also justified for the purely damped radiative mode (Equation (13)). However, including gravity on small scales introduces a qualitatively different behavior to the fourth mode, which is purely damped on scales larger than the Jeans length and purely unstable on scales smaller than the Jeans length.

Keeping the field equations, but again ignoring terms $\propto \dot{a} / a$, $\propto \dot{\Phi}$, and the equations for the matter, I find

$$
\begin{aligned}
\omega^{4} & +i \dot{\tau} \omega^{3}\left[1+\frac{1}{R}+\frac{8}{27 \dot{\tau}^{2}}\left(k^{2}+\xi_{r}^{2}\right)\right] \\
& -\omega^{2}\left[\frac{k^{2}}{3}\left(1+\frac{8}{9 R}\right)-\frac{\xi_{r}^{2}}{27 R}\left(9 R^{2}+R-8\right)\right] \\
& -i \dot{\tau}\left(\frac{1+R}{R}\right) \omega\left[\frac{k^{2}}{3(1+R)}-\frac{\xi_{r}^{2}}{3}(1+R)-\frac{8 k^{2} R^{2} \xi_{r}^{2}}{81 \dot{\tau}^{2}(1+R)}\right] \\
& -\frac{1}{9} k^{2} R \xi_{r}^{2}=0,
\end{aligned}
$$

where $\xi_{r}^{2}=16 \pi G a^{2} \rho_{r}$. As before, there are four orthogonal modes admitted by Equation (14) The first and second are gravity- and radiative-diffusion-modified acoustic modes. To first order in $\dot{\tau}^{-1}$ these are

$$
\begin{aligned}
\omega_{\text {acoustic }} & = \pm\left[c_{s}^{2} k^{2}-\xi_{r}^{2} \frac{(1+R)}{3}\right]^{1 / 2}+\frac{i}{\dot{\tau}}\left(1-\frac{(1+R) \xi_{r}^{2}}{3 c_{s}^{2} k^{2}}\right)^{-1} \\
& \times\left[\frac{-k^{2}}{2(1+R)}\left(c_{s}^{2} R^{2}+\frac{8}{27}\right)+\frac{4 \xi_{r}^{2}}{27}\left(R+\frac{(1+R) \xi_{r}^{2}}{3 c_{s}^{2} k^{2}}\right)\right] .
\end{aligned}
$$

This expression should be compared with Equation (12). Note that taking either the $k \rightarrow \infty$ or the $\xi_{r}^{2} \rightarrow 0$ limits in Equation (15) recovers Equation (12). Furthermore, the first term in Equation (15) is identical to what one would expect for the Jeans instability. Setting the first term in square brackets to zero, I derive the Jeans length:

$$
\frac{\lambda_{J}}{2 \pi}=\left(\frac{k_{J}}{a}\right)^{-1}=\left(\frac{3 c_{s}^{2}}{16 \pi G \rho_{r}(1+R)}\right)^{1 / 2} .
$$

The third mode admitted by Equation (14) is the strongly damped diffusion mode, $\omega_{\text {damped }}$, of Equation (13), which is unmodified by the inclusion of gravity to second order in $\dot{\tau}^{-1}$. The fourth and final mode admitted by Equation (14) is purely imaginary. It is

$$
\omega_{\mathrm{KH}} \approx \frac{i k^{2}}{9 \dot{\tau}}\left(\frac{R^{2}}{1+R}\right)\left(\frac{\xi_{r}^{2}}{c_{s}^{2} k^{2}-\xi_{r}^{2}(1+R) / 3}\right)
$$

to first order in $\dot{\tau}^{-1}$. This expression follows from equating the last two terms in Equation (14). Note that in the limit that the medium is Jeans stable $c_{s}^{2} k^{2}>(1+R) \xi_{r}^{2} / 3$ (large comoving wavenumber, small spatial scale) Equation (17) implies that the medium is unstable. Conversely, on scales larger than $\lambda_{\mathrm{J}}, \omega_{\mathrm{KH}}$ is damped. In the high- $k$ limit $\left(c_{s}^{2} k^{2} \gg(1+R) \xi_{r}^{2} / 3\right)$, Equation (17) can be written simply as

$$
\frac{\omega_{\mathrm{KH}}}{a} \approx i \frac{R^{2} \xi_{r}^{2}}{3 \dot{\tau} a}=-i \frac{3 \pi G}{\kappa_{T} c}\left(\frac{\rho_{b}}{\rho_{r}}\right),
$$


which is independent of spatial scale. Precisely when the medium is stable to the classical Jeans instability, it is unstable to a slow diffusive mode.

The characteristic frequency is smaller than that for any of the other modes: the timescale for growth is $\left[3 \pi G /\left(\kappa_{T} c\right)\right]^{-1} \approx$ $2 \times 10^{16} \mathrm{~s}$, much longer than the radiation acoustic timescale, $\omega_{\text {acoustic }}^{-1} \sim\left(c_{s} k\right)^{-1}$, on a scale of the order of the Hubble radius and the interaction timescale $\omega_{\text {damped }}^{-1}$. The subscript "KH" is used to emphasize the connection with the KelvinHelmholtz timescale for gravitational contraction of a radiation pressure supported self-gravitating body (see Section 2.2). The timescale $t_{\mathrm{KH}}$ is roughly $m_{e} / m_{p}$ shorter than the timescale for the radiative instability derived by Gamow (1949) and Field (1971) $\left(\sim 10^{12} \mathrm{yr}\right.$; their Equations (20) and (58), respectively), and does not formally require a temperature difference between the radiation field and the gas (see Section 3).

\subsection{The Simplest Case with Dark Matter}

The slow diffusive mode derived above changes when dark matter is included primarily because the strength of the potential changes. In the WKB limit, the mode structure becomes more complicated when the Euler and continuity equations for the dark matter are included and the dispersion relation is cumbersome. Nevertheless, neglecting $\dot{a} / a, \dot{\Phi}$, and neutrinos as before, the slow diffusive mode can be isolated in the dispersion relation. To first order in $\dot{\tau}^{-1}$, it reads

$$
\omega_{\mathrm{KH}} \approx i \frac{k^{2}}{3 \dot{\tau}}\left(\frac{R}{1+R}\right)\left[\frac{\xi_{b}^{2}+\xi_{m}^{2}(1+8 /(9 R))}{c_{s}^{2} k^{2}-\xi_{r}^{2}(1+R) / 3-\xi_{m}^{2}}\right],
$$

where $\xi_{b, m, r}^{2}=16 \pi G a^{2} \rho_{b, m, r}$. Compare Equation (19) with Equation (17). Again taking the strongly Jeans stable limit of small spatial scales and large $k\left(c_{s}^{2} k^{2} \gg \xi_{m}^{2}+\xi_{r}^{2}(1+R) / 3\right)$, I have that

$$
\omega_{\mathrm{KH}} \approx i \frac{R \xi_{m}^{2}}{\dot{\tau}}\left[\frac{\rho_{b}}{\rho_{m}}+1+\frac{8}{9 R}\right],
$$

In the limit of small $R$ this expression becomes

$$
\frac{\omega_{\mathrm{KH}}}{a} \approx-i \frac{128 \pi G}{9 \kappa_{T} c}\left(\frac{\rho_{m}}{\rho_{b}}\right) .
$$

As in Equation (18), this timescale is independent of spatial scale and it does not evolve with the scale factor. As long as the medium is diffusive (the tight-coupling approximation holds) and one consider scales smaller than the Jeans length, the medium is unstable. Furthermore, note that taking instead the large $R$ limit in Equation (20) recovers the basic form of Equation (18), but with the substitution $\rho_{b} \rightarrow \rho_{b}+\rho_{m}$ in the numerator. However, for $z>1000, R<1$ and Equation (21) is the relevant limit. The characteristic growth timescale is then

$$
t_{\mathrm{KH}} a \sim \frac{a}{\omega_{\mathrm{KH}}} \approx \frac{9 \kappa_{T} c}{128 \pi G}\left(\frac{\rho_{b}}{\rho_{m}}\right) \sim 4 \times 10^{14}\left(\frac{10 \rho_{b}}{\rho_{m}}\right) \mathrm{s} .
$$

Note the correspondence with the Salpeter timescale for black hole growth. The characteristic timescale in Equation (22) can be understood as the timescale for a region of differential volume to radiate away its total (relativistic) thermal energy content $\left(e \sim \rho_{b} c^{2}\right)$ at the Eddington limit $\left(\dot{e} \sim 4 \pi G \rho_{\text {tot }} c / \kappa_{T}\right):^{3}$

$$
t_{\mathrm{KH}} \sim \frac{e}{\dot{e}} \sim \frac{c \kappa_{T}}{4 \pi G}\left(\frac{\rho_{b}}{\rho_{\mathrm{tot}}}\right) .
$$

\footnotetext{
3 The "9" in Equations (21) and (22) originates in the numerical factor in Equation (3) connecting $\Theta_{1}$ and $\Theta_{2}$ (see, e.g., Hu \& Sugiyama 1996).
}

This is simply the $\mathrm{KH}$ timescale. $t_{\mathrm{KH}}$ can equivalently be thought of as the ratio of the square of the dynamical timescale, $\left(4 \pi G \rho_{\mathrm{tot}}\right)^{-1}$, to the photon-baryon interaction timescale, $\left(\kappa_{T} \rho_{b} c\right)^{-1}$. Alternatively, one may think of the growth timescale as $t_{\mathrm{KH}}=t_{\text {diff }}\left(t_{\mathrm{dyn}} / t_{\mathrm{r}}\right)^{2}$, where $t_{\text {diff }}=c k^{2} /\left(3 \kappa_{T} \rho_{b}\right)$ is the diffusion time, $t_{\mathrm{dyn}}=\left(4 \pi G \rho_{\mathrm{tot}}\right)^{-1 / 2}$ is the dynamical time, and $t_{\mathrm{r}}=\left(c_{s} k\right)^{-1}$ is the radiation acoustic sound-crossing timescale on a scale $k^{-1}$. Finally, one can also obtain this characteristic timescale by equating the frictional force between the radiation and the fluid due to Thomson scattering with the gravitational force. The timescale for instability is $\sim m_{e} / m_{p}$ shorter than the timescale for the instability discussed in Gamow (1949) (see also Field 1971). Because the mode that becomes unstable has $\omega=0$ in the adiabatic limit, one may identify this unstable mode as the entropy mode (e.g., Lithwick \& Goldreich 2001).

Importantly, $t_{\mathrm{KH}}$ in Equation (22) is roughly 100 times the age of the universe at decoupling and approximately 500 times the age of the universe at radiation-matter equality. At earlier times the instability also operates, but because $t_{\mathrm{KH}}$ is a constant in the large- $k$ limit, it becomes increasingly long compared to the Hubble time at higher redshift. This is most clearly seen by writing

$$
t_{\mathrm{KH}} H(z) a \sim \frac{1}{\Omega_{m}}\left(\frac{\Gamma}{H(z)}\right),
$$

where $\Gamma$ is the interaction timescale and $H$ is the Hubble parameter. Because $\Gamma \gg H(z)$ in the tight-coupling limit, $t_{\mathrm{KH}} H(z) a$ is much larger than unity, and the instability is slow.

For a non-relativistic discussion of this unstable mode and its potential astrophysical applications, see Kaneko \& Morita (2006) and Thompson (2008). The same mode has been discussed by Yamamoto et al. (1998), where they term it the "terminal velocity mode" (their Section 3.3 ), since one can write the baryon velocity as $v_{b} \simeq k \Psi R / \dot{\tau}$ (their notation) by equating the gravitational force with the drag force associated with the coupling to the radiation field. As I have shown above, the mode is unstable at arbitrarily large (but, not infinite) $\dot{\tau}$, with fixed growth timescale, and operates until decoupling. As Yamamoto et al. (1998) show, the instability smoothly joins the pressure-less collapse solution after decoupling.

Note that physically equivalent modes should operate whenever the medium is supported against self-gravity by a particle species that diffuses. For example, in the epoch before neutrinomatter decoupling at a time $t_{H} \sim 1 \mathrm{~s}$ and at a temperature larger than $\sim 10^{10} \mathrm{~K}$ a similar mode is expected to have growth rate of order

$$
\frac{\omega}{a} \sim-i \frac{4 \pi G \rho_{m}}{\Gamma}
$$

where here $\rho_{m}$ is the mass density in all matter, and $\Gamma \sim G_{F}^{2} T^{5} / \hbar$ is the weak interaction rate; $\Gamma \approx 0.2 T_{\mathrm{MeV}}^{5} \mathrm{~s}^{-1}$ where $T_{\mathrm{MeV}}=$ $T / \mathrm{MeV}$. The mass density in matter at $z \sim 10^{10}$ is of order $\rho_{m} \approx 1 \mathrm{~g} \mathrm{~cm}^{-3}$ and so I find that $(\omega / a) \sim-i 10^{-6} \mathrm{~s}^{-1}$. That is, the growth timescale is of order one million times the age of the universe at that epoch.

\section{SLOW GRAVITATIONAL INSTABILITY IN AN EXPANDING BACKGROUND}

The preceding analysis approximated the background state as fixed. However, the growth timescale derived exceeds the timescale for the background state to change by a factor of more than 100. This invalidates the WKB calculation and necessitates a treatment with an evolving background. This is most simply 
treated on small scales where the gravitational potential may be approximated as Newtonian. For simplicity of presentation, I treat the non-relativistic case with $a T^{4} \ll \rho c^{2}$ and I neglect dark matter. In this case, the Eulerian equations governing the evolution in an inertial frame are (e.g., Silk 1967)

$$
\begin{gathered}
\left(\frac{\partial \rho}{\partial t}\right)_{\mathbf{r}}+\nabla_{\mathbf{r}} \cdot(\rho \mathbf{u})=0 \\
\left(\frac{\partial \mathbf{v}}{\partial t}\right)_{\mathbf{r}}+\mathbf{u} \cdot \nabla \mathbf{u}=-\frac{1}{\rho} \nabla_{\mathbf{r}} p-\nabla_{\mathbf{r}} \Phi \\
\left(\frac{\partial e}{\partial t}\right)_{\mathbf{r}}+\mathbf{u} \cdot \nabla_{\mathbf{r}} e+\frac{4}{3} e \nabla_{\mathbf{r}} \cdot \mathbf{u}=-\nabla_{\mathbf{r}} \cdot \mathbf{F}, \\
\nabla^{2} \Phi=4 \pi G \rho,
\end{gathered}
$$

and

$$
\mathbf{F}=-\frac{c}{3 \kappa_{T} \rho} \nabla_{\mathbf{r}} e .
$$

Here, $\rho$ is the mass density of gas, $\mathbf{u}$ is the velocity, and $p=a T^{4} / 3$ and $e=3 p$ are the radiation pressure and energy density, respectively. The gas and radiation are assumed to have the same temperature, and it is assumed that $p$ greatly exceeds $p_{g}=n k_{B} T$, the gas pressure. ${ }^{4}$

In Equations (26)-(30), I have adopted the notation of Peebles (1993) (Chapter 5). The subscript $\mathbf{r}$ reflects reference to an inertial coordinate system. Transforming to a comoving coordinate system with $\mathbf{x}=\mathbf{r} / a(t)$ and $\mathbf{u}=\dot{a} \mathbf{x}+\mathbf{v}(\mathbf{x}, t)=$ $\dot{a} \mathbf{x}+a \dot{\mathbf{x}}$, where $\mathbf{v}$ is the peculiar velocity, and writing $\rho=\rho(t)(1+$ $\delta(\mathbf{x}, t))$, with $\rho(t) \propto a(t)^{-3}$, and taking $\ddot{a} / a=-(4 / 3) \pi G \rho(t)$, Equations (26)-(30) become

$$
\begin{gathered}
\frac{\partial \delta}{\partial t}+\frac{1}{a} \nabla \cdot[(1+\delta) \mathbf{v}]=0, \\
\frac{\partial \mathbf{v}}{\partial t}+\frac{\dot{a}}{a} \mathbf{v}+\frac{1}{a} \mathbf{v} \cdot \nabla \mathbf{v}=-\frac{1}{a \rho(1+\delta)} \nabla p-\frac{1}{a} \nabla \phi, \\
\frac{\partial e}{\partial t}+\frac{1}{a} \nabla \cdot(e \mathbf{v})+4 \frac{\dot{a}}{a} e+\frac{p}{a} \nabla \cdot \mathbf{v}=\frac{c}{3 \kappa \rho a^{2}} \nabla \cdot\left[\frac{1}{(1+\delta)} \nabla e\right], \\
\nabla^{2} \phi=4 \pi G a^{2} \delta,
\end{gathered}
$$

where time and space derivatives are now understood to be in the comoving frame and $\Phi=\phi(\mathbf{x}, t)+(2 / 3) \pi G \rho(t) a^{2} x^{2}$. In analogy with the density perturbation I write $e=e(t)(1+\varepsilon(\mathbf{x}, t))$, and Equation (33) can be rewritten as

$$
\frac{\partial \varepsilon}{\partial t}+\frac{1}{a} \nabla \cdot[(1+\varepsilon) \mathbf{v}]+\frac{1+\varepsilon}{3 a} \nabla \cdot \mathbf{v}=\frac{c}{3 \kappa \rho a^{2}} \nabla \cdot\left[\frac{\nabla \varepsilon}{1+\delta}\right] .
$$

So far the equations written are completely general for the problem at hand. I now specialize to the case of small departures from the background state so that terms of order $\delta|v|, \delta \varepsilon$, and $\varepsilon|v|$ can be neglected. The resulting equations can be written as

$$
\frac{\partial^{2} \delta}{\partial t^{2}}+2 \frac{\dot{a}}{a} \frac{\partial \delta}{\partial t}-4 \pi G \rho \delta=\frac{e}{3 \rho a^{2}} \nabla^{2} \varepsilon
$$

and

$$
\frac{\partial \varepsilon}{\partial t}-\frac{4}{3} \frac{\partial \delta}{\partial t}=\frac{c}{3 \kappa \rho a^{2}} \nabla^{2} \varepsilon
$$

\footnotetext{
4 For the general case with gas pressure, see Thompson (2008).
}

Note that the left-hand side of Equation (37) is simply

$$
\frac{\partial \varepsilon}{\partial t}-\frac{4}{3} \frac{\partial \delta}{\partial t}=\frac{4}{3} \frac{\partial}{\partial t}\left(\frac{\Delta s}{s}\right)
$$

where $s$ is the total entropy. Writing $\varepsilon=\Delta e / e=4 \Delta T / T$, Equations (36) and (37) become identical to Equations (1) and (2) of Silk (1967). Expanding these expressions in Fourier components with wavenumber $\tilde{k}=k / a$, taking $a(t) \propto t^{2 / 3}$, and defining the temperature perturbation as $q=\varepsilon / 4$, results in two coupled ordinary differential equations for the time evolution of the density and temperature perturbations:

$$
\ddot{\delta}_{\tilde{k}}+\frac{4}{3 t} \dot{\delta}_{\tilde{k}}-\frac{2}{3 t^{2}} \delta_{\tilde{k}}+3 c_{r}^{2} \tilde{k}^{2} q_{\tilde{k}}=0
$$

and

$$
\dot{q}_{\tilde{k}}-\frac{1}{3} \dot{\delta}_{\tilde{k}}+\tilde{\omega} q_{\tilde{k}}=0
$$

where over-dots denote time derivatives,

$$
\tilde{\omega}=\frac{c \tilde{k}^{2}}{3 \kappa \rho}
$$

is the diffusion rate,

$$
c_{r}^{2}=\frac{4}{9} \frac{e}{\rho}=\left.\frac{\partial p}{\partial \rho}\right|_{s}
$$

is the adiabatic radiation pressure sound speed, and the subscript $\tilde{k}$ has been added to emphasize that these equations are for the evolution of the density and temperature, $\delta$ and $q$, at a specific comoving wavenumber.

\subsection{Adiabatic Modes}

In the adiabatic limit $\tilde{\omega}=0$ in Equation (40), $\partial / \partial t(\Delta s / s)=0$, $q=(1 / 3) \delta$, and the system becomes (e.g., Peebles Equation (5).124)

$$
\ddot{\delta}_{\tilde{k}}+\frac{4}{3 t} \dot{\delta}_{\tilde{k}}+\left(c_{r}^{2} \tilde{k}^{2}-\frac{2}{3 t^{2}}\right) \delta_{\tilde{k}}=0 .
$$

In the long-wavelength limit $(\tilde{k} \rightarrow 0)$ one recovers the classic equation for the time evolution of the density contrast, modeled as a purely pressure-less fluid, which has the growing and damped solutions $\delta_{+} \propto t^{2 / 3}$ and $\delta_{-} \propto t^{-1}$, respectively. Taking $\tilde{k} \rightarrow \infty$, Equation (43) describes an acoustic wave, damped by the expansion of the universe.

\subsection{Non-Adiabatic Evolution}

The full non-adiabatic evolution is more complicated. First, as shown by Silk (1967) and others, the acoustic modes are damped by radiative diffusion. Second - the purpose of this paper-as shown in the previous sections, it is formally incorrect to neglect gravity at large comoving wavenumber, because to do so is to effectively set the driving term for the diffusive gravitational instability to zero.

To illustrate the importance of these effects, I solve Equations (39) and (40) for $\delta_{\tilde{k}}(t)$ and $q_{\tilde{k}}(t)$ with $\dot{\delta}_{\tilde{k}}(t=0)=0$, $\delta_{\tilde{k}}(t=0)=1, q_{\tilde{k}}(t=0)=1 / 3, c_{r}^{2} \tilde{k}^{2}=100 / t^{2 / 3}$, and $\tilde{\omega}=t^{2} / \sqrt{10}$ over the range $0.1 \leqslant t \leqslant 100$. These parameters ensure that the mode considered is much smaller than the Jeans scale and that the diffusion rate is small. Figure 1 shows 

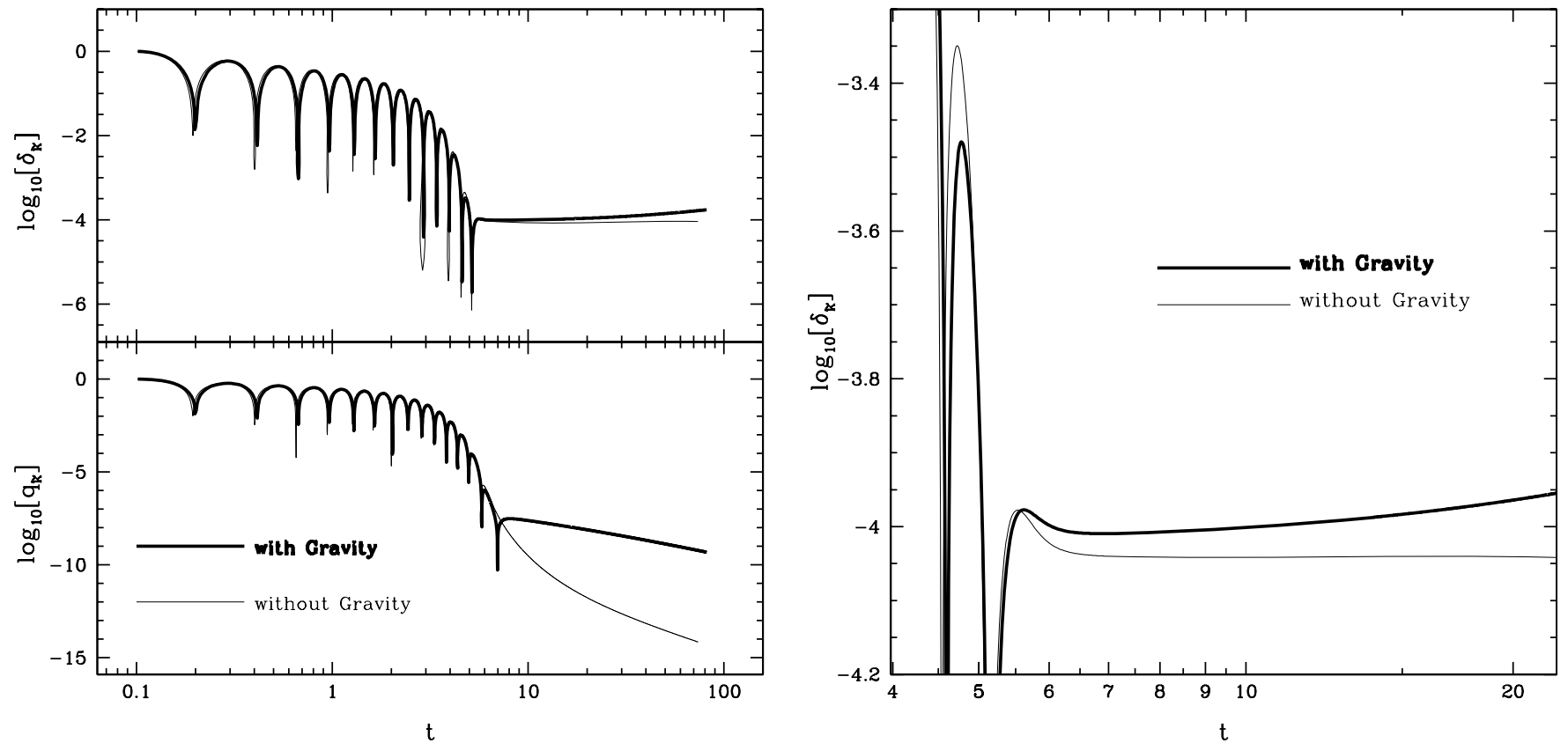

Figure 1. Left panel: solution to Equations (39) and (40) at large $\tilde{k}$ as a function of time both with gravity (the " $2 / 3 t^{2}$ " term; heavy solid line) and without gravity (light solid). The top and bottom panels show the evolution of the density and temperature perturbations, $\delta_{\tilde{k}}(t)$ and $q_{\tilde{k}}(t)$. The acoustic wave is rapidly damped by both the expansion of the universe and diffusive damping. At early times, the two solutions track each other closely. After the mode is damped, the evolution is qualitatively different as a result of the slow diffusive mode. Right panel: a zoom in on the late-time evolution of $\delta_{\tilde{k}}$ to highlight the difference between the calculations with and without gravity. The increase in $\delta_{\tilde{k}}(t)$ at late times is not a result of the breakdown of the tight-coupling approximation, but is instead a result of the instability highlighted in this paper. Importantly, in a more complete calculation the medium becomes optically thin, the radiation decouples, and the solution joins the classic pressure-less free-fall collapse solution. See Yamamoto et al. (1998) and Singh \& Ma (2002) for detailed calculations at small scales.

results for the time evolution of the density and temperature perturbations with and without gravity (heavy and light solid lines, respectively). The acoustic mode is damped by both the expansion of the universe and Silk damping. At early times, the evolution with and without gravity is very similar. At later times, the presence of the unstable growing mode becomes more pronounced and the evolution of $\delta_{\tilde{k}}$ and $q_{\tilde{k}}$ is qualitatively different. The right panel shows a zoomed-in version of the late-time evolution of $\delta_{\tilde{k}}$. At $t \approx 6$, as the mode becomes fully damped, the fractional difference in $\delta_{\tilde{k}}$ between the two solutions is a few to $10 \%$. Of course, this example does not include the physics of decoupling of the mode and gravitational free-fall into the dark matter potential, which would be included in a full calculation, and so the difference between the two solutions becomes exaggerated at late times (see Yamamoto et al. 1998; Singh \& Ma 2002).

\section{DISCUSSION}

The original treatments of acoustic modes near the epoch of radiation-matter equality focused on the importance of radiative damping of acoustic modes at large comoving wave number. The general equations derived by Silk (1967) (and Silk 1968; Peebles \& Yu 1970; Weinberg 1971) contain the physics described above, but the slow diffusive mode these equations admit was not discussed because gravity was neglected when calculating the damping of acoustic modes on small scales. This approximation eliminated the unstable diffusive mode. Similarly, the work of Hu \& Sugiyama (1996) provides a concise analytic treatment of acoustic mode damping, but again neglects the importance of gravity at large comoving wavenumber. For the unstable mode I have highlighted, the $k$-dependence drops out in the high$k$ limit because of the $k$-dependence of the diffusion timescale.
Thus, to first order in the limit of large $\dot{\tau}$ it is not consistent with neglect gravity at high- $k$. Yamamoto et al. (1998) have discussed the same instability, but it is important to emphasize that the timescale for the mode's growth is a constant on small scales as long as the tight coupling approximation is valid (Equation (22)). In this way, the mode's existence is not a manifestation of the breakdown of the tight coupling approximation; even at very high $\dot{\tau}$, the instability exists with fixed $t_{\mathrm{KH}} a$.

Most importantly, on all scales smaller than the Jeans length and at all times before decoupling, the medium is unstable, albeit on a long timescale. Thus, it is not formally correct to think of the medium on scales below the horizon as stable. Although it is dynamically stable, it is unstable on the $\mathrm{KH}$ timescale for a radiation pressure supported self-gravitating medium $\left(\sim \kappa_{T} c / G\right)$. The instability should operate at all scales smaller than the Jeans length for which the medium is tightly coupled. The physics of the mode is simply that self-gravitating radiation pressure supported media radiate their total internal energy content at the Eddington limit. It is qualitatively different than the mode described by Gamow (1949) and Field (1971).

The physics of this mode is already contained in the popular codes CMBFAST and CAMB for calculating the growth of perturbations in the tight-coupling limit. However, precision analytic and numerical studies of primordial perturbations in density and temperature that neglect gravity on small scales may find systematic small differences with respect to more complete calculations that are attributable to the growth of this mode (e.g., in the transfer function (e.g., Yamamoto et al. 1998), or the BAO scale, or in the initial conditions for the formation of very small scale dark matter halos).

As noted in Section 2, the instability identified in Equation (22) should operate at essentially all times before decoupling because it depends only on the ratio $\left(\rho_{m} / \rho_{b}\right)$ and 
the opacity $\kappa_{T}$. However, because the growth timescale is constant, it becomes increasingly long compared to the age of the universe at earlier times (see Equation (24)). Thus, one expects it to be most important at the latest times for which the assumptions apply: the largest Jeans-stable modes at a time near decoupling. Even here, the growth timescale is much longer than the age of the universe, as can be seen from Equation (24). More generally, the medium should be unstable to analogous modes any time it is supported against self-gravity by the radiation pressure provided by a diffusing particle species (Section 2.2).

I thank Chris Hirata for a number of useful conversations, as well as David Spergel, Matias Zaldarriaga, Lam Hui, Scott Dodelson, and Martin White for discussions. I am additionally grateful to the Aspen Center for Physics, where a portion of this work was completed, and to D. Zifkin for encouragement. This work is supported in part by an Alfred P. Sloan Fellowship.

\section{REFERENCES}

Blaes, O., \& Socrates, A. 2003, ApJ, 596, 509

Dodelson, S. 2003, Modern Cosmology (Amsterdam, Netherlands: Academic) Field, G. B. 1971, ApJ, 165, 29

Gamow, G. 1949, Reviews of Modern Physics, 21, 367

Hu, W., \& Sugiyama, N. 1995a, ApJ, 444, 489

Hu, W., \& Sugiyama, N. 1995b, Phys. Rev. D, 51, 2599

Hu, W., \& Sugiyama, N. 1996, ApJ, 471, 542

Hu, W., Sugiyama, N., \& Silk, J. 1997, Nature, 386, 37

Kaneko, N., \& Morita, K. 2006, Ap\&SS, 305, 349

Lithwick, Y., \& Goldreich, P. 2001, ApJ, 562, 279

Ma, C.-P., \& Bertschinger, E. 1995, ApJ, 455, 7

Peebles, P. J. E. 1993, Princeton Series in Physics (Princeton, NJ: Princeton Univ. Press)

Peebles, P. J. E., \& Yu, J. T. 1970, ApJ, 162, 815

Silk, J. 1967, Nature, 215, 1155

Silk, J. 1968, ApJ, 151, 459

Singh, S., \& Ma, C.-P. 2002, ApJ, 569, 1

Thompson, T. A. 2008, ApJ, 684, 212

Weinberg, S. 1971, ApJ, 168, 175

Yamamoto, K., Sugiyama, N., \& Sato, H. 1998, ApJ, 501, 442 